\title{
Standardization of Bivariate Flow Karyotypes of Human Chromosomes for Clinical Applications
}

\author{
Hideki Kuriki ${ }^{1 *}$ and Hakuo Takahashi ${ }^{2}$ \\ ${ }^{1}$ Nippon Becton Dickinson Company, Ltd; \\ ${ }^{2}$ Department of Clinical Sciences and Laboratory Medicine, Kansai Medical University, Osaka, Japan
}

\begin{abstract}
Flow cytometry can be used for the detection of aberrations in DNA composition and DNA content of human chromosomes. We demonstrate here the magnitude of DNA content differences among normal chromosomes of 20 healthy individual donors and the clinical applications of the standardized bivariate flow karyotype. Peak positions were determined from Hoechst $33258(\mathrm{HO})$ VS Chromomycin A3 (CA3) flow karyotypes of individuals, and the values of the relative chromosomal DNA content were normalized to the peak position constituting chromosomes $9-12$, to which a value of 100 arbitrary units (a.u.) was assigned. The mean values and standard deviations that were $\mathrm{HO}$ and CA3 fluorescence intensity for each
\end{abstract}

chromosome were calculated. The range of standard deviation is 1.43 to 6.21 (CA3) and is 1.22 to $4.28(\mathrm{HO})$. The standard deviations for each chromosome did not overlap; therefore, it was decided that the standardized bivariate flow karyotype was suitable to use for clinical applications. Further study using fluorescence in situ hybridization for chromosome 22 that was observed for extra peak accompanied with normal peak in the flow karyotype of clinical material did not suggest more positive data. This suggests that the peak-position difference between chromosome 22 homologues may be a result of a combination of satellite and heterochromatic-region size differences. J. Clin. Lab. Anal. 11:169-174, 1997. @1997Wiley-Liss, Inc.

Key words: flow cytometry; standardize; Hoechst $33258(\mathrm{HO})$; chromomycine A3 (CA); chromosome 22; FISH

\section{INTRODUCTION}

Flow cytometry is useful for clinical applications of flow chromosome analysis and can be used for detecting aberrations in DNA composition and DNA content of human chromosomes (1-4). For chromosome analysis on the human genetics and the medical genetics, we have used classical chromosome banding techniques: GTG, Q, R, C, and Ag-banding. Additionally, fluorescence in situ hybridization (FISH) of human chromosomes has become a valuable tool for detecting chromosome aberrations and for the mapping of congenital genetic disease or positioning of tumor genes. In the methods described above, there have been some problems and limitations that have required several days of banding analysis to obtain the necessary results. Even then, the DNA aberrations are not always detected clearly. Recently, new FISH products, which use simple methods and take a short time of preparation, have been developed.

Flow karyotypes of single chromosomes were reported $(5,6)$ in 1975. A flow cytometer equipped with a single laser was commonly used for flow karyotype analysis and the sorting of chromosomes. Following the development of single laser flow cytometry, flow cytometers equipped with dual lasers were used to obtain high precision chromosome analysis and for sorting of human chromosomes. Owing to this development, some chromosome anomalies were detected by using flow cytometry for the first time.

By microscopic chromosome banding analysis, it was known that the frequency of some chromosome variants differs among ethnic populations and from person to person $(7,8)$. It was demonstrated that chromosomal aberrations using bivariate fluorescence distributions were capable of detecting $(9,10)$. The most heteromorphic chromosomes were $\mathrm{Y}, 21$, $22,15,16,13,14$, and 19 , and chromosomes $2-8$ and $X$ were least heteromorphic (9). The base composition of the variable regions differed among chromosome types (9) and the variants on DNA sequences were laid on through repetitive DNA regions that it was heterochromatic regions.

On the cytogenetic analysis of cell biology, we have used now the microscopic analysis (e.g., GTG, Q, C, and Ag-banding). We investigated the flow karyotype instead of conventional banding methods. The standard pattern of healthy donor's flow karyotype was obtained, and differences in peak

*Correspondence to: Hideki Kuriki, 11-1, 2-chome, Nakamichi, Higashinariku, Osaka-city, Osaka 537, Japan.

Received 9 October 1996; Accepted 26 October 1996 
position between homologous chromosomes and DNA contents were analyzed. The marker chromosome, observed for extra peak position in the flow karyotype of clinical material, was painted by FISH painting.

\section{MATERIALS AND METHODS}

\section{Cell Culture}

Human leukocytes were obtained from the peripheral blood of 49 healthy donors 11-47 years, 27 males and 22 females, using Lymphoprep ${ }^{\mathrm{TM}}$ (NYCOMED, Oslo, Norway). They were cultured for $72 \mathrm{hr}$ in RPMI 1640 medium (Gibco BRL Products, Life Technologies, Grand Island, NY) supplemented with $15 \%$ fetal calf serum (Gibco), $100 \mathrm{U} / \mathrm{ml}$ penicillin, 100 $\mathrm{mg} / \mathrm{ml}$ streptomycin, $2.5 \mathrm{mg} / \mathrm{ml}$ fungizone, and phytohemagglutinin P (Difco Lab, Detroit, MI) in $25 \mathrm{~cm}^{2}$ tissue culture flask. Cells were cultivated at $37^{\circ} \mathrm{C}$ in a humidified atmosphere of $5 \% \mathrm{CO}_{2}$ in air.

\section{Chromosome Isolation}

Cultivated lymphocytes were blocked in mitosis by adding colcemid $(0.1 \mathrm{mg} / \mathrm{ml})$ for $12-16 \mathrm{hr}$. The lymphocytes were transferred into the $15 \mathrm{ml}$ centrifugal tube and centrifuged at $120 \times \mathrm{G}$ for $5 \mathrm{~min}$. The cell pellets were resuspended in swelling buffer $(75 \mathrm{mM} \mathrm{KCl})$, and afterward the cells were resuspended in Polyamine-Digitonin isolation buffer $(15 \mathrm{mM}$ Tris-HCl, 2 mM EDTA, $80 \mathrm{mM} \mathrm{KCl,} 20 \mathrm{mM} \mathrm{NaCl}, 0.5 \mathrm{mM}$ EGTA, 400 mM Spermine, $1 \mathrm{M}$ Spermidine). Pool cells plus isolation buffer were vortexed vigorously for 10-30 sec and stored on ice for at least $10 \mathrm{~min}$.

\section{Chromosome Staining}

In order to keep large clumps and nuclei to a minimum, chromosome suspensions were centrifuged at $30 \times \mathrm{G}$ for $1 \mathrm{~min}$, and $1 \mathrm{ml}$ of supernatant was removed to the new tube. Chromosomes were stained with $150 \mu \mathrm{l}$ of Chromomycin A3 (CA3) fluorochrome (Wako Pure Chemical Industries, Osaka, Japan), final CA3 concentration $55 \mu \mathrm{M}$, and stored on ice or in refrigerator for $3 \mathrm{hr}$ to overnight. Immediately before analyzing on the flow cytometer, $4.5 \mu \mathrm{l}$ of Hoechst 33258 (HO) fluorochrome (Wako) was added (final HO concentration was $3.6 \mu \mathrm{M}$ ).

\section{Flow Cytometric Analysis}

Chromosomes were analyzed using the FACS Vantage flow cytometer (Becton Dickinson Immunocytometry Systems, San Jose, CA) equipped with Consort 32 software and two Argon ion lasers (Spectra Physics, Mountain View, CA). One laser was tuned to emit light in the UV (351-364 nm, $200 \mathrm{~mW}$ ) for exciting $\mathrm{HO}$ fluorescence, and the second laser was tuned to emit light at $457 \mathrm{~nm}(150 \mathrm{~mW})$ for exciting CA3 fluorescence. HO intensity excited with UV was analyzed after passing through a $400 \mathrm{~nm}$ long pass filter. CA3 fluorescence resulting from excitation by the $457 \mathrm{~nm}$ was analyzed after passing through a $520 \mathrm{~nm}$ long pass filter.

Orientation and spacing of the laser beams were adjusted, using $2.0 \pm 0.02 \mu \mathrm{m}$ Fluoresbrite ${ }^{\mathrm{TM}}$ yellow-green microspheres (Polysciences, Northampton, UK). The adjustment was such that the detection of the emitted light of UV light excited microspheres in the $\mathrm{HO}$ channel and the emitted light in the CA3 channel on excitation with $457 \mathrm{~nm}$ light was optimized to produce the lowest coefficient of variation $(\mathrm{CV})$ possible $(<1.2 \%)$. Chromosome suspensions were made to flow through the nozzle using FACS Flow sheath solution (Becton Dickinson) that was azide free. The flow rate for analysis was 100-200 events per sec.

\section{Data Analysis}

For each flow karyotype, 10,000 chromosomes were measured. The number of chromosome in each peak was used to determine the weight of each peak. The center of each peak was calculated using the method of least-squares that was included in the FACS Consort 32 application programs. The values for $\mathrm{HO}$ fluorescence and CA3 fluorescence, that the total sum of the peak positions of all autosomal chromosomes except 9-12 and sex chromosomes was divided by 18 (the value was the chromosome peak number), were normalized by setting the mean of the HO fluorescence and of the CA3 fluorescence of all chromosome peaks at values of 100 arbitrary units (a.u.).

For examination of the accuracy of the flow cytometer, the means and standard errors (SEs) of the distances from chromosome 1 to another chromosome were calculated for individuals on the values of $\mathrm{HO}$ fluorescence and CA3 fluorescence.

\section{Fluorescence In Situ Hybridization}

Metaphase slides were prepared from cultured lymphocytes and were hybridized using a slightly modified method described by Takahashi et al. (11) with a biotinylated specific DNA probe Coatasome 22 (Oncor, Gaithersburg, MD). Sites of hybridization were fluorescently labeled with fluorescent isothiocyanate (FITC)-conjugated avidin. Chromosomes were counterstained with propidium iodide (PI). Photomicrographs were made with a fluorescence microscope using Kodak Echtachrome color slide film (ASA 400).

\section{RESULTS}

\section{Experimental Chromosome Variation}

In the flow karyotypes of 49 phenotypically normal donors, 29 flow karyotypes showed a polymorphism. The polymorphic phenomena were mostly observed for chromosomes 13-22 and Y. The peripheral blood lymphocytes were prepared using the same methods so the polymorphic phenomena did not depend on method of preparation. The flow karyotype in this investigation had two types of polymorphic 
phenomenon, one that showed two peaks on a certain chromosome and the other demonstrating a different peak position of a certain chromosome. The former depends on how the chromosomes are prepared and the setting on the flow cytometer, and the latter depends on DNA differences between the maternal and paternal homologues for chromosomes. Figure 1 shows the CA3 and HO fluorescence intensities of chromosomes isolated from a peripheral blood lymphocyte culture of a healthy female donor. In this flow karyotype, the polymorphisms can be observed for chromosomes 17, 21, and 22 . On the spontaneously transformed human B-lymphoblastoid cell line GM00130B, each chromosome dot area converged narrowly, and no polymorphisms for any chromosome was observed (data not shown).

\section{Determination of Standard Flow Karyotype}

To obtain the standard flow karyotype, the peak position coordinates were determined for each homologue in each karyotype and were normalized as described in Material and Methods. The range of observed peak positions is shown in Figure 2. Each dot represents the peak position of one chromosome homologue from one of the individuals. For the large chromosomes (1-12 and X) each chromosome dot pattern except chromosome 1 and 4, converged narrowly. For the smaller chromosomes (13-22 and Y) the chromosome dot pattern (especially chromosome 19 and 22) spread slightly. Moreover, for chromosome 14, 15, 16, and 17, the HO axis values of peak positions were closely located and had a tendency to overlap on the a.u. histogram. For each peak in the

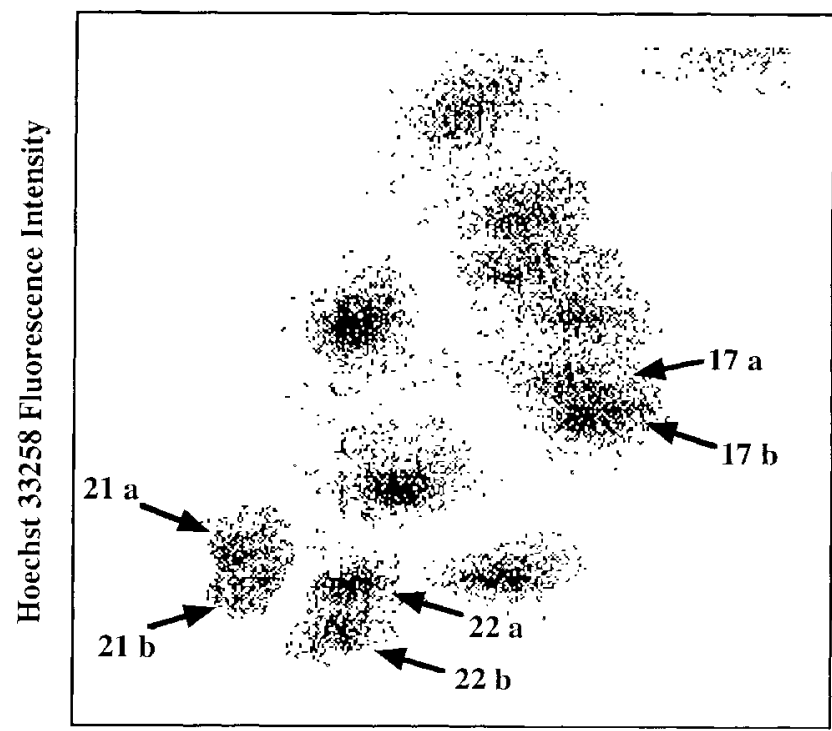

Chromomycin A3 Fluorescence Intensity

Fig. 1. Bivariate flow karyotype of isolated mitotic chromosomes of the phenotypical normal donor. It shows the partial karyotype (chromosome 13-22). The specific peaks in the flow karyotype are labeled to identify the chromosome. bivariate flow karyotype, the mean values of CA3 and $\mathrm{HO}$ fluorescence and their standard deviations (SD) were determined from the calculated a.u. data of all 20 samples collectively. The mean positions and the range of standard deviations are shown in Figure 3a. The maximal standard deviations of CA3 and HO fluorescence of all 20 samples were 6.21 and 4.28 that were shown on chromosome 1 . The smallest values were 1.43 (CA3 of chromosome Y) and 1.22 (HO of chromosome 18). The each standard deviation range did not overlap for each chromosome.

The maximal coefficient of variation determined from 20 independent samples, was $8.8 \%$ (CA3 fluorescence of chromosome 22) and 5.6\% (HO fluorescence of chromosome 22 and $\mathrm{Y}$ ).

\section{Calculation of Standard Errors for Examination of Accuracy}

For the analysis of flow karyotypes, peak positions showed only a slight change with every repeated analysis, because it was difficult to establish a tight internal standard. When a slight discrepancy from standard peak position was noted in the flow karyotype, it was determined to be either a congenital variation or analytical error. Each standard error value is shown in Table 1 . The maximal standard errors of CA3 and HO were 2.40 and 1.56. These data showed that the flow cytometer used in this investigation was holding steady and the flow data acquired by the flow cytometer were reliable.

The above noted results indicate that Figure $3 \mathrm{~b}$ is accepted as a standard flow karyotype of human chromosomes.

TABLE I. Means and Standard Errors of Peak-Position Interval of Twenty Donors

\begin{tabular}{lrrrrr}
\hline & \multicolumn{2}{c}{ CA3 } & & \multicolumn{2}{c}{ HO } \\
\cline { 2 - 3 } \cline { 5 - 6 } Interval no. & Mean & SE & & Mean & SE \\
\hline$\Delta 2$ & 21.93 & 1.52 & & -2.55 & 1.04 \\
$\Delta 3$ & 63.03 & 1.78 & & 20.95 & 1.11 \\
$\Delta 4$ & 85.31 & 2.40 & & 19.82 & 1.23 \\
$\Delta 5$ & 79.07 & 2.03 & & 34.17 & 1.10 \\
$\Delta 6$ & 84.55 & 2.12 & & 41.78 & 1.14 \\
$\Delta 7$ & 83.15 & 1.55 & 54.96 & 1.12 \\
$\Delta 8$ & 100.50 & 1.85 & & 64.13 & 1.23 \\
$\Delta 9-12$ & 101.44 & 1.66 & & 75.75 & 1.11 \\
$\Delta 13$ & 135.58 & 1.79 & 85.74 & 1.16 \\
$\Delta 14$ & 127.04 & 1.35 & & 98.36 & 1.12 \\
$\Delta 15$ & 126.28 & 1.50 & 103.93 & 1.07 \\
$\Delta 16$ & 123.26 & 1.03 & & 114.09 & 1.40 \\
$\Delta 17$ & 121.34 & 1.14 & 122.07 & 1.11 \\
$\Delta 18$ & 152.29 & 1.88 & 111.57 & 1.17 \\
$\Delta 19$ & 135.73 & 1.22 & 139.85 & 1.14 \\
$\Delta 20$ & 147.00 & 1.49 & 129.99 & 1.22 \\
$\Delta 21$ & 169.86 & 1.64 & 139.80 & 0.98 \\
$\Delta 22$ & 154.31 & 1.07 & 144.85 & 1.13 \\
$\Delta \mathrm{X}$ & 99.94 & 2.11 & 55.34 & 1.22 \\
$\Delta \mathrm{Y}$ & 183.43 & 1.93 & 119.70 & 1.56 \\
\hline & & & &
\end{tabular}




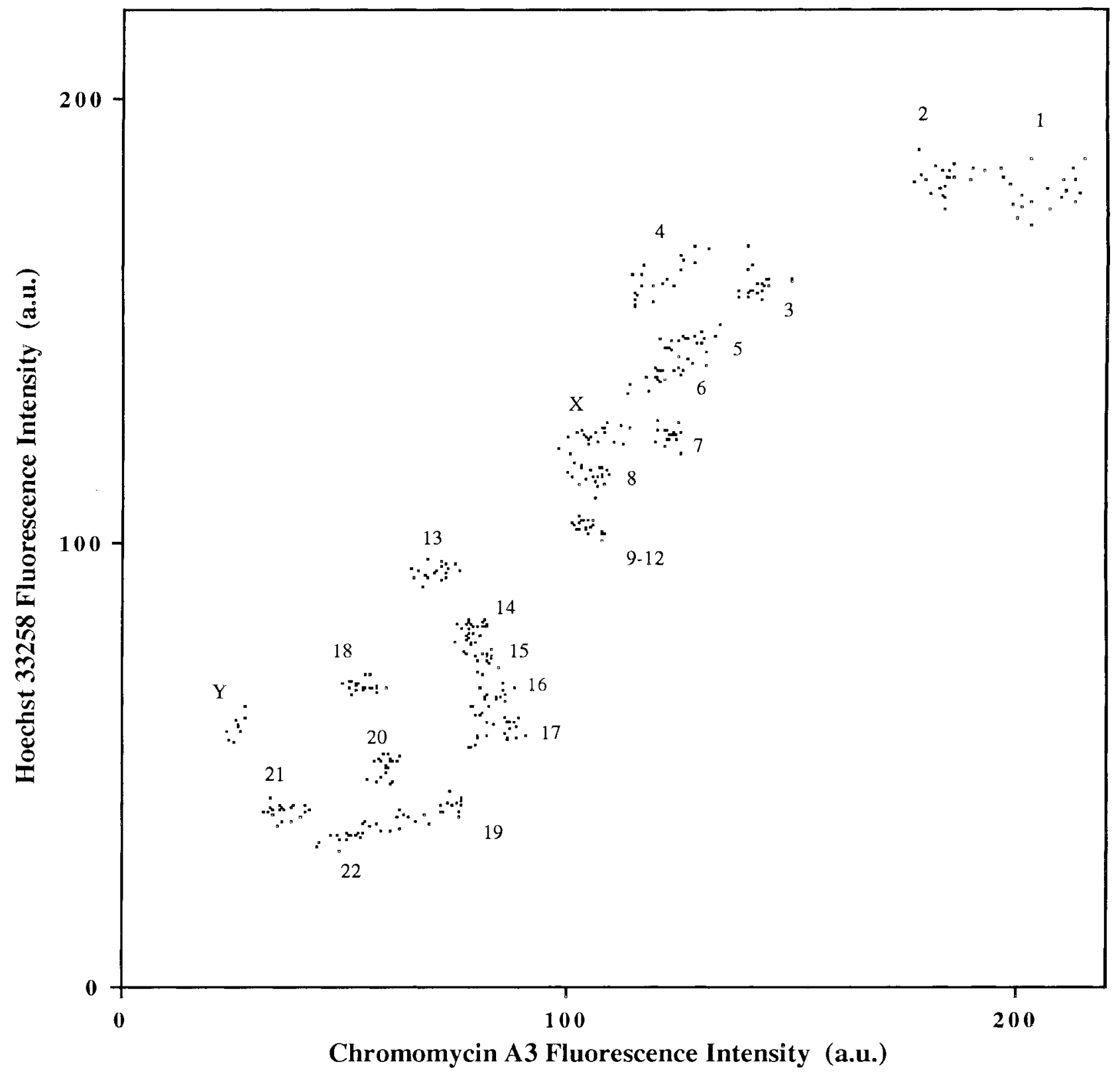

Fig. 2. The flow karyotype peak position variability in normal chromosomes of 20 phenotypical normal unrelated individuals. Each point represents the position of a measured homologue. Peak positions were normalized

\section{Chromosome Painting by Fluorescence In Situ Hybridization}

Figure 4 shows metaphase spreads after fluorescence in situ hybridization. The hybridized slides were analyzed by a fluorescence microscope and photographed. Whole chromosome 22 was painted with Coatasome 22 probe. The specific fluorescence of FITC-conjugated avidin was weak, but identification of chromosome 22 was confirmed. The homologous chromosome 22 was wholly stained, and no chromosomal structural abnormalities were found. In this case, the heteropolymorphism observed on the flow karyotype was not by setting the mean of the CA3 and HO fluorescence of all chromosome peaks at values of 100 arbitrary units (a.u.) in each karyotype.

based on the congenital chromosomal abnormality, but on the DNA heteropolymorphism.

\section{DISCUSSION}

The present study demonstrates that the standard pattern of flow karyotype can be used as clinical cytogenetic screening for genetic counseling. It is important that any chromosomal anomaly is detected quickly for genetic counseling, and the precise flow karyotype of a client is valuable on a clinical diagnostic. It is possible to diagnose a disease certainly by using a flow karyotype with polymerase chain reac- 


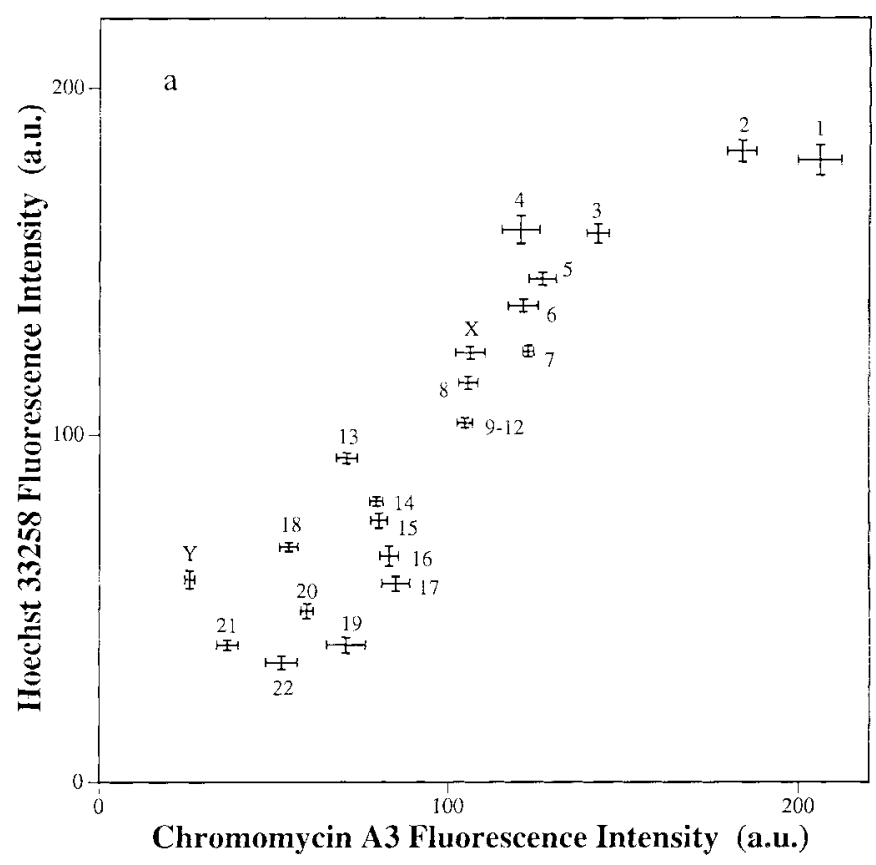

Fig. 3. The total variation in peak positions of chromosomes was calculated for 20 unrelated individuals. (a) The cross points of vertical and horizontal line show a mean CA3 fluorescence intensity (a.u.) and HO

tion (PCR) and FISH. The chromosomal anomaly associated with malignant tumor cells is often complex, based on the extra peak positions observed on the flow karyotype. For analyzing and sorting abnormal chromosomes from malignant

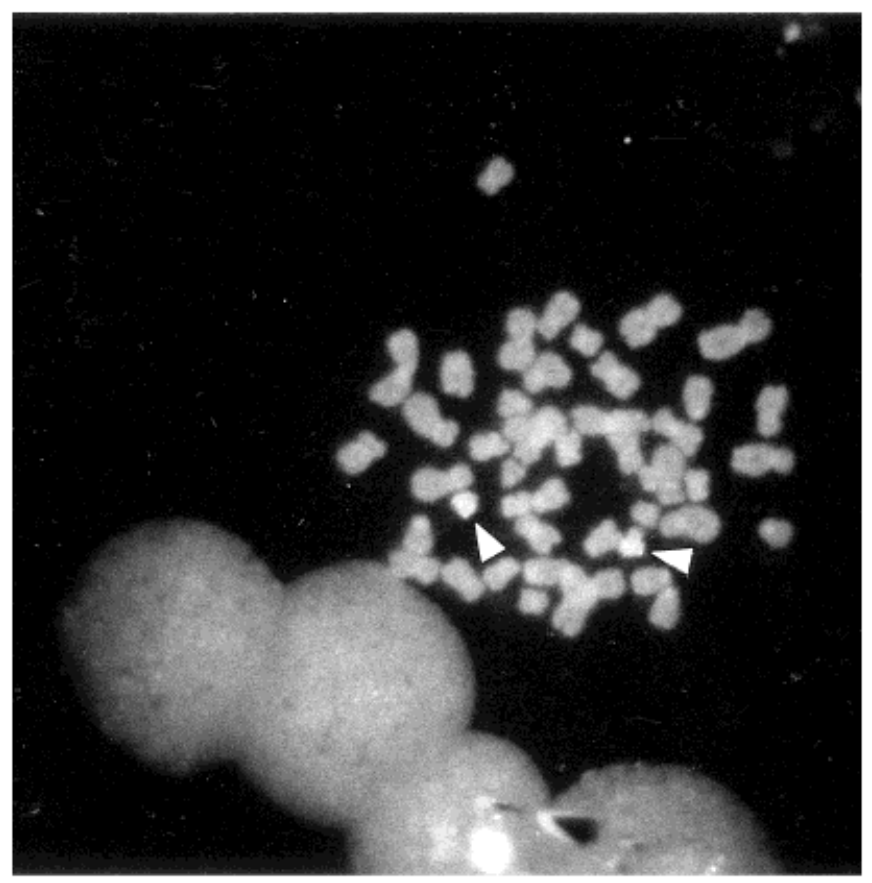

Fig. 4. Fluorescence in situ hybridization with chromosome specific probe Coatasome 22. Minor cross hybridization of weak to medium intensity was observed, as indicated by arrows.

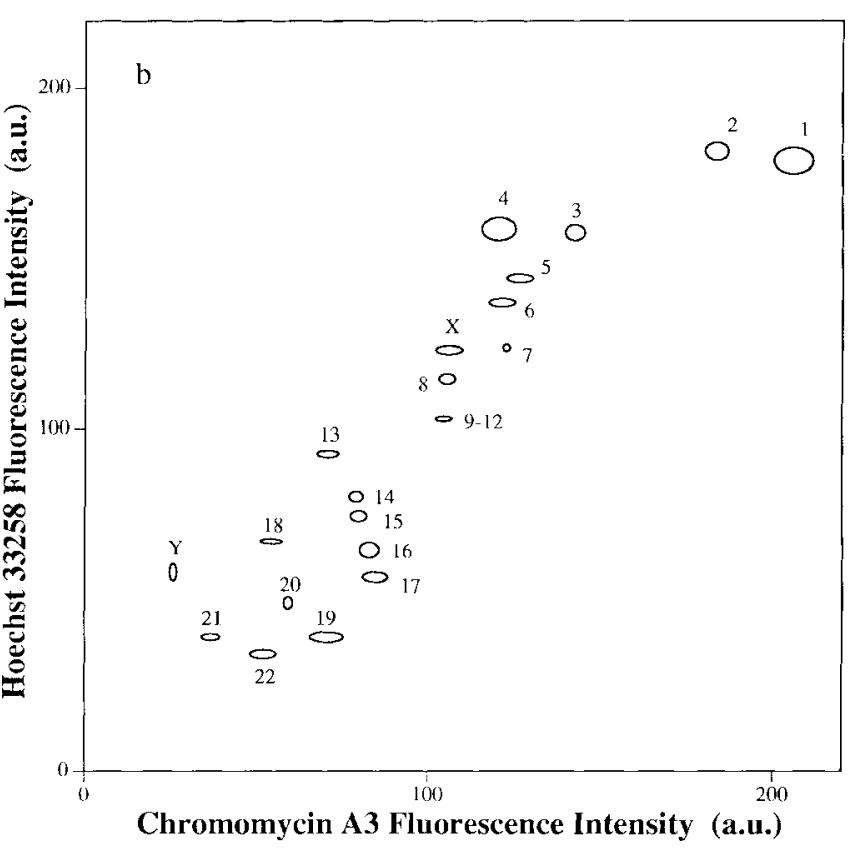

fluorescence intensity (a.u.). The ranges show value of mean \pm 1 SD. (b) The circles and ellipses were made from the data shown in (a), they appear in the area of the standard flow karyotype.

tumor cells, extra peaks must be well separated from the normal peaks on the flow karyotype. For this method to work for detecting small aberrations, the extent of experimental variation must be monitored (10).

The normalized data for each chromosome pair were tested for statistical evidence of homologous differences. To determine whether heteromorphic differences in flow karyotype peak position can be interpreted as differences in the DNA content of variant chromosomes, the DNA content estimates from flow karyotype peak positions were compared with those made by the CYDAC flying-spot cytometric microscope system $(9,10,13)$. The DNA contents of the mitotic chromosomes from 10 children and their parents in four families were quantified by bivariate flow karyotypes, and the measured absolute difference in homologue DNA content between children and their parents averaged $0.8 \%$, or $\approx 1 \mathrm{Mbp}$ over all chromosome types (12). Trask et al. (9) demonstrated that meiotic recombination within large tracts of repetitive sequences in heterochromatin or in acrocentric satellites may be suppressed relative to meiotic recombination in the rest of the genome. Bivariate flow karyotypes of individuals revealed heteromorphisms in chromosomes 1,16 , and $\mathrm{Y}$ of $8 \%, 16 \%$, and $30 \%$, respectively.

For conventional chromosome analysis using $\mathrm{R}, \mathrm{Q}$, and GTG-banding, an average chromosome band has a DNA length of $\sim 6 \mathrm{Mbp}$ per band. It is therefore possible to detect chromosomal anomalies only in cases where a chromosomal anomaly appears on a large band. Moreover, chromosomal heteropolymorphism was able to detect on the conventional 
chromosomal analysis. For example, the size of the centromeric region stained by $\mathrm{C}$-banding differed from each other in homologous chromosome 1 , and variation of satellite size of acrocentric chromosomes was detected by GTG-banding. These variations appeared on a flow karyotype as the heteropolymorphism of each chromosomal peak position on a flow karyotype. The small DNA variation (about several hundred Kbp), however, can be detected on a flow karyotype because the DNA variation is reflected by the fluorescence intensity of the CA3 and HO fluorochromes.

\section{ACKNOWLEDGMENTS}

The authors thank Mr. Hiromitsu Shimada for fluorescence in situ hybridization staining work and Dr. L. Scott Cram for his advice and help in correcting the manuscript.

\section{REFERENCES}

1. Harris P, Boyd E, Young BD, Ferguson-Smith MA: Determination of the DNA content of human chromosomes by flow cytometry. Cytogenetic Cell Genet 41:14-21, 1986.

2. Deaven LL, Van Dilla MA, Bartholdi MF, CarranoAV, Cram LS, Fuscoe JC, Gray JW, Hildebrand CE, Moyzis RK, Perlman J: Construction of human chromosome specific DNA libraries from flow-sorted chromosomes. Quant Bio LI:159-167, 1986.

3. Lebo RV, Anderson LA, Lau YFC, Flandermeyer R, Kan YW: Flowsorting analysis of normal and abnormal human genomes. Quant Bio LI:169-176, 1986.
4. Manogaran PS, Pande G: Identification of a marker chromosome in a rat ascitic tumor by flow cytometry using a modified method for preparing chromosome suspensions. Cytometry 14:349-353, 1993.

5. Stubblefield E, Cram LS, Deaven L: Flow microfluorometric analysis of isolated Chinese hamster chromosomes. Exp Cell Res 94:464468, 1975.

6. Gray JW, Carrano AV, Steinmentz LL, van Dilla MA, Moore DH, Mayall BH, Mendelsohn ML: Chromosome measurement and sorting by flow systems. Proc Natl Acad Sci USA 72:1231-1234, 1975.

7. Lubs HA, Ruddle FH: Chromosome polymorphism in American Negro and white populations. Nature 233:134-136, 1971.

8. Lubs HA, Kimberling WJ, Hecht R, Patil SR, Brown J, Gerald P, Summitt RL: Racial differences in the frequency of $\mathrm{Q}$ and $\mathrm{C}$ chromosomal heteromorphisms. Nature 268:631-633, 1977.

9. Trask B, van den Engh G, Mayall B, Gray JW: Chromosome heteromorphism quantified by high-resolution bivariate flow karyotyping. Am J Hum Genet 45:739-752, 1989.

10. Boschman GA, Rens W, van Oven CH, Manders EMM, Aten JA: Bivariate flow karyotyping of human chromosomes: evaluation of variation in Hoechst 33258 fluorescence, Chromomysin A3 fluorescence, and relative chromosomal DNA content. Cytometry 12:557-569, 1991

11. Takahashi E, Hori T, Lawrence JB, McNell J, Singer RH, O'Connell P, Leppert M, White R: Human type II collagen gene (COL2A1) assigned to chromosome $12 \mathrm{q} 13.1$ or q13.2 by in situ hybridization with biotinylated DNA probe. Jpn J Hum Genet 34:307-311, 1989.

12. Trask B, van den Engh G, Gray W: Inheritance of chromosome heteromorphisms analyzed by high-resolution bivariate flow karyotyping. Am J Human Genet 45:753-760, 1989.

13. Mayall BH, Carrano AV, Moore II DH, Ashworth LK, Bennett DE, Mendelsohn ML: The DNA-based human karyotype. Cytometry 5:376385,1984 . 